CLINICAL STUDY

\title{
Analysis of expression and function of the inhibitory receptor ILT2 in lymphocytes from patients with autoimmune thyroid disease
}

\author{
Lesly Doníz-Padilla $^{1,2}$, Amalia E Paniagua ${ }^{2}$, Pilar Sandoval-Correa ${ }^{3}$, Adriana Monsiváis-Urenda ${ }^{1}$, \\ Susanna Leskela ${ }^{2}$, Mónica Marazuela ${ }^{2}$ and Roberto González-Amaro ${ }^{1}$ \\ ${ }^{1}$ Department of Immunology, School of Medicine, Universidad Autónoma de San Luis Potosí, Avenue V. Carranza 2405, \\ 78210 San Luis Potosí, S.L.P., Mexico, ${ }^{2}$ Service of Endocrinology and ${ }^{3}$ Unit of Molecular Biology, Hospital Universitario de la Princesa, \\ Universidad Autónoma de Madrid, C/Diego de León 62, 28006 Madrid, Spain \\ (Correspondence should be addressed to R González-Amaro; Email: rgonzale@uaslp.mx; M Marazuela; Email: mmarazuela.hlpr@salud.madrid.org)
}

\begin{abstract}
Objective: Autoimmune thyroid disease (AITD) is characterized by different defects in immunoregulatory mechanisms. The immunoglobulin-like transcript receptor 2 (ILT2) or leukocyte Ig-like receptor 1 (LIRB1/CD85j) exerts an important immunoregulatory role. We hypothesized that the lymphocytes from AITD patients have a diminished expression and function of ILT2. The aim of this study was to investigate the expression and function of ILT2 in lymphocytes from patients with AITD. Design and methods: In this study, 18 patients with Hashimoto's thyroiditis (HT), 20 with Graves' disease, and 26 healthy controls were studied. ILT2 expression was analyzed by flow cytometry and immunohistochemistry in peripheral blood mononuclear cells (PBMC) and thyroid tissue. The regulatory function of ILT2 was assessed by an assay of inhibition of lymphocyte proliferation and by an analysis of cell cycle progression. The effect of ILT2 on cytokine synthesis was also evaluated. Results: We found a significant increased expression of ILT2 by lymphocytes in AITD patients. ILT2 was also detected in the leukocyte infiltrate of thyroid tissue from HT patients. On the contrary, a significant diminished inhibitory activity of ILT2 on cell proliferation was observed in AITD patients. In addition, PBMC from AITD patients showed a diminished synthesis of interleukin 10 on ILT2 engagement. Conclusions: The abnormal expression and function of ILT2 detected in AITD suggests that this receptor may participate in the pathogenesis of this condition.
\end{abstract}

European Journal of Endocrinology 165 129-136

\section{Introduction}

The activation of lymphocytes requires a complex balance between positive and negative signals that are generated as a result of recognition phenomena between ligands and receptors during the interaction of these lymphocytes with antigen presenting cells (1). The integration of these signals ensures that $\mathrm{T}$ lymphocyte activation occurs only in time and right place. When activation signals prevail, an efficient immune response is generated, whereas when inhibitory signals predominate, anergy or immune tolerance is observed (1).

Co-receptor stimulation and negative regulation of $\mathrm{T}$ lymphocytes have widely been characterized in the case of CD28 and CTLA4 (CD152), respectively, which interact with CD80 and CD86 (2). Signaling through the TCR and $\mathrm{CD} 28$ promotes proliferation and differentiation of T cells, whereas the interaction of CTLA4 with their ligands generates inhibitory signals. Inhibition of
$\mathrm{T}$ cell activation is a key phenomenon in peripheral tolerance, which is of great importance in the pathogenesis of autoimmune diseases (3). Other molecules are also able to suppress the activation of T cells including the leukocyte Ig-like receptors (LIRs) or immunoglobulin-like transcript receptors (ILTs) (4). At least, ten genes encode this family of receptors, which recognize class I major histocompatibility complex molecules. Inhibitory ILT receptors bear a long cytoplasmic tail that contains tyrosine inhibitory motifs (ITIMs), which recruit the Src homology-containing protein-tyrosine phosphatases SHP1 and SHP2 (4).

ILT2 (LIRB1, CD85j) is expressed on various proportions of NK cells $(23-77 \%), \mathrm{CD} 8+\mathrm{T}$ lymphocytes (10-36\%), and CD $4+$ T cells (5-19\%) as well as by all B lymphocytes, monocytes, and bone marrow-derived dendritic cells (5). It has been described that HLA-G as well as some HLA-A, HLA-B, and HLA-C alleles and the UL18 gene product of cytomegalovirus specifically interact with ILT2 (6). This receptor inhibits the 
cytotoxic function of NK cells and increases the activation threshold of CD8 + cytotoxic T lymphocytes (7). In CD $4+\mathrm{T}$ cells, the cross-linking of ILT2 inhibits the proliferation and production of interleukin 2 (IL2) and interferon $\gamma$, although inducing the synthesis of IL10 and transforming growth factor $\beta$ (8). Furthermore, this receptor inhibits calcium mobilization in B lymphocytes, the respiratory burst in monocytes, and the immunogenic function of dendritic cells $(9,10)$.

Autoimmune diseases result from the failure of the immune system to develop tolerance toward selfantigens. The autoimmune thyroid diseases (AITD) include a number of conditions, which have the generation of cellular or humoral immune responses against the thyroid gland in common (11). AITD are relatively common, affecting more than 5\% of the general population, and as most autoimmune diseases occur primarily in women by a ratio of 9:1 (12). AITD can be divided into two major subtypes, Hashimoto's thyroiditis (HT) and Graves' disease (GD) (12). Although the etiology of these conditions has not been elucidated, different factors seem to contribute to their pathogenesis including polymorphisms of CTLA4 and CD4O genes, the presence of HLA-B8, HLA-DR3, HLA-DR4, and HLA-DR 5 alleles, environmental factors, stress, and cytokines (13-15). In addition, we have previously described that AITD patients show a diminished function and levels of $\mathrm{T}$ regulatory (Treg) cells and also an enhanced synthesis of Th17 cytokines $(16,17)$. Furthermore, AITD patients exhibit increased levels of angiogenic molecules, which likely contribute to the inflammatory phenomenon $(18,19)$.

HT is characterized by massive infiltration of the thyroid gland by immune cells, leading to its destruction. Most patients have autoantibodies to thyroglobulin (Tg) and thyroperoxidase (TPO) (20). On the other hand, GD usually shows a small inflammatory cell infiltrate and its main immunological feature is the presence of autoantibodies directed against the TSH receptor, which stimulate the growth and function of thyroid follicular cells, thus leading to the development of hyperthyroidism (21).

The role of ILT2 in the pathogenesis of different conditions has been described including mycobacterial and HIV infection $(22,23)$. In addition, we have previously explored the expression and function of this immunomodulatory receptor in lymphocytes from patients with systemic lupus erythematosus (24). Given the regulatory role of ILT2 in the activation and proliferation of lymphocytes and the immune dysregulation observed in AITD, we decided to explore the possible role of this receptor in patients with HT and GD. Our results indicate that patients with AITD show an abnormal expression and function of ILT2 and suggest that this regulatory receptor may contribute to the immunopathogenesis of thyroid autoimmunity.

\section{Materials and methods}

\section{Patients and samples}

A total of 38 AITD patients that fulfilled the diagnostic criteria for AITD were studied (18 with HT and 20 with GD). Peripheral blood samples were obtained from all patients, and a complete thyroid work-up was performed on them including history and physical examination. In this study, 26 healthy subjects with similar gender and age to patients were studied as controls. Thyroid gland specimens were obtained from four patients with HT and one patient with GD who had relapsed after antithyroid drug treatment and were euthyroid under carbimazole therapy at the time of surgery. Thyroid tissue was maintained in medium until processing. In all cases, a written informed consent was obtained, and the local hospital bioethics committee approved this study. All studies were carried out in accordance with the principles set out in the declaration of Helsinki.

\section{Cells}

Peripheral blood mononuclear cells (PBMC) were isolated by Ficoll-Hypaque (Sigma Chemical Co.) gradient centrifugation. Cells were resuspended in RPMI 1640 culture medium (Hyclone, Logan, UT, USA) supplemented with $10 \%$ fetal bovine serum (Hyclone), $2.0 \mathrm{mM}$ glutamine, 50 units $/ \mathrm{ml}$ penicillin, and $50 \mu \mathrm{g} / \mathrm{ml}$ streptomycin (Sigma). To isolate thyroid mononuclear cells (TMC), thyroid specimens were minced and digested with collagenase $(1.0 \mathrm{mg} / \mathrm{ml}$; Roche Molecular Biochemicals) in Hank's balanced
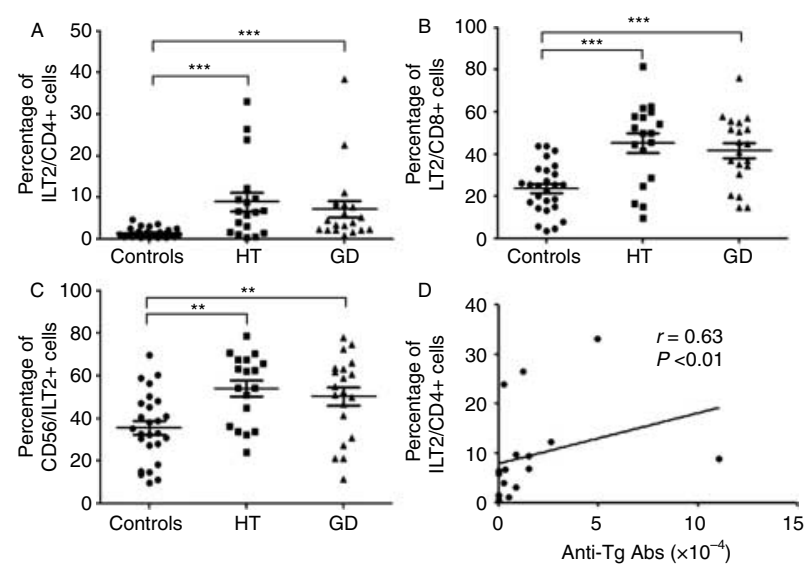

Figure 1 Expression of ILT2 in patients with AITD and their correlation with autoantibody levels. (A-C) PBMC from patients with Hashimoto's thyroiditis (HT), Graves' disease (GD), and healthy controls were analyzed by flow cytometry for the expression of ILT2 in $\mathrm{CD} 4+(\mathrm{A}), \mathrm{CD} 8+(\mathrm{B})$, and CD56 + (C) cells. Data correspond to the arithmetic mean and S.E.M. ${ }^{* *} P<0.01$ and ${ }^{* * *} P<0.001$, ANOVA, or Kruskal-Wallis test. (D) Correlation analysis between the expression of ILT2 by CD4 + cells and the serum levels of anti-Tg autoantibodies. Correlation coefficient and $P$ value are shown. 
salt solution (BioWhittaker, Lonza, Verviers, Belgium) for $1 \mathrm{~h}$ at $37^{\circ} \mathrm{C}$. Then, cells were passed through a steel mesh, and mononuclear cells were isolated by FicollHypaque centrifugation. Finally, TMC were washed twice with PBS and resuspended in complete RPMI 1640 culture medium. Cell viability was always higher than $95 \%$.

\section{Antibodies}

The following MAB were used for flow cytometry analyses: anti-CD4, anti-CD8, anti-CD14, anti-CD19, and anti-CD56 (all labeled with FITC and obtained from BD Biosciences (San Jose, CA, USA)). A biotinylated anti-ILT2 MAB (HP-F1, R\&D Systems, Minneapolis, MN, USA) and streptavidin labeled with phycoerythrin (PE, BD Biosciences) were also employed. For histological analyses, anti-CD4, anti-CD8, anti-CD20, anti-CD57, and anti-CD68 MAB (Master Diagnóstica, Granada, Spain) were used.

\section{Flow cytometry analysis}

Cells were immunostained with a biotinylated anti-ILT2 MAB followed by avidin conjugated with PE. Then, cells were incubated with anti-CD4, anti-CD8, anti-CD14, anti-CD19, or anti-CD56 MAB labeled with FITC. Cells were washed with PBS and fixed in 1\% paraformaldehyde and analyzed in a FACSCalibur flow cytometer (Becton Dickinson, San Jose, CA, USA), using the Cellquest Software (Becton Dickinson).

\section{Cell proliferation assays}

PBMC $\left(5.0 \times 10^{5}\right)$ were loaded with $5.0 \mathrm{mM}$ carboxyfluorescein diacetate succinimidyl ester (Molecular Probes, Eugene, OR, USA). Then, cells were cultured for $72 \mathrm{~h}$ in flat bottom 96-well plates previously coated with an anti-CD3 (10 $\mu \mathrm{g} / \mathrm{ml}$, Biolegend, San Diego, CA, USA) and an anti-CD28 MAB (10 $\mu \mathrm{g} / \mathrm{ml}$, Immunotech, Marseille, France), in the presence or absence of HP-F1 anti-ILT2 MAB plus a rabbit anti-mouse IgG (Sigma), as a cross-linker reagent. Finally, cells were analyzed by flow cytometry and results were expressed as the percent of divided cells. In some cases, anti-CD4-PE or anti-CD8PE (BD Biosciences) antibodies were used to analyze the cell proliferation in these lymphocyte subsets. These results were expressed as the percent of divided cells.

\section{Cell cycle analysis}

Cell cycle analysis was performed by a DNA content assay propidium iodide staining and flow cytometry. Briefly, cells $\left(5.0 \times 10^{5}\right)$ were poured in 96-well flat bottom plates, stimulated with $10 \mu \mathrm{g} / \mathrm{ml}$ anti-CD3 and anti-CD28, and cultured in the presence or absence of anti-ILT2, HP-F1 MAB plus a rabbit anti-mouse IgG. After $72 \mathrm{~h}$ of culture, cells were harvested and stained for $30 \mathrm{~min}$ with $30 \mu \mathrm{g} / \mathrm{ml}$ propidium iodide (Sigma) and $0.5 \mathrm{mg} / \mathrm{ml}$ RNAse (Sigma) at $4{ }^{\circ} \mathrm{C}$ and analyzed in a FACSCalibur flow cytometer.

\section{Immunohistochemistry}

Tissue specimens were fixed and embedded in paraffin. Serial tissue sections 3-4 mm thick were mounted on glass slides (DakoCytomation, Copenhagen, Denmark) and heated and treated with the real target retrieval solution containing citrate buffer pH 6.0 (Dako, Glostrup, Denmark) to expose hidden antigens. Slides were also pre-treated with Real Peroxidase-Blocking Solution (Dako) to block the endogenous peroxidase. The Dako REAL EnVision Detection System, Rabbit/ Mouse (Dako) was used to detect the indicated primary MAB. Finally, sections were counterstained with Carazzi's hematoxylin.
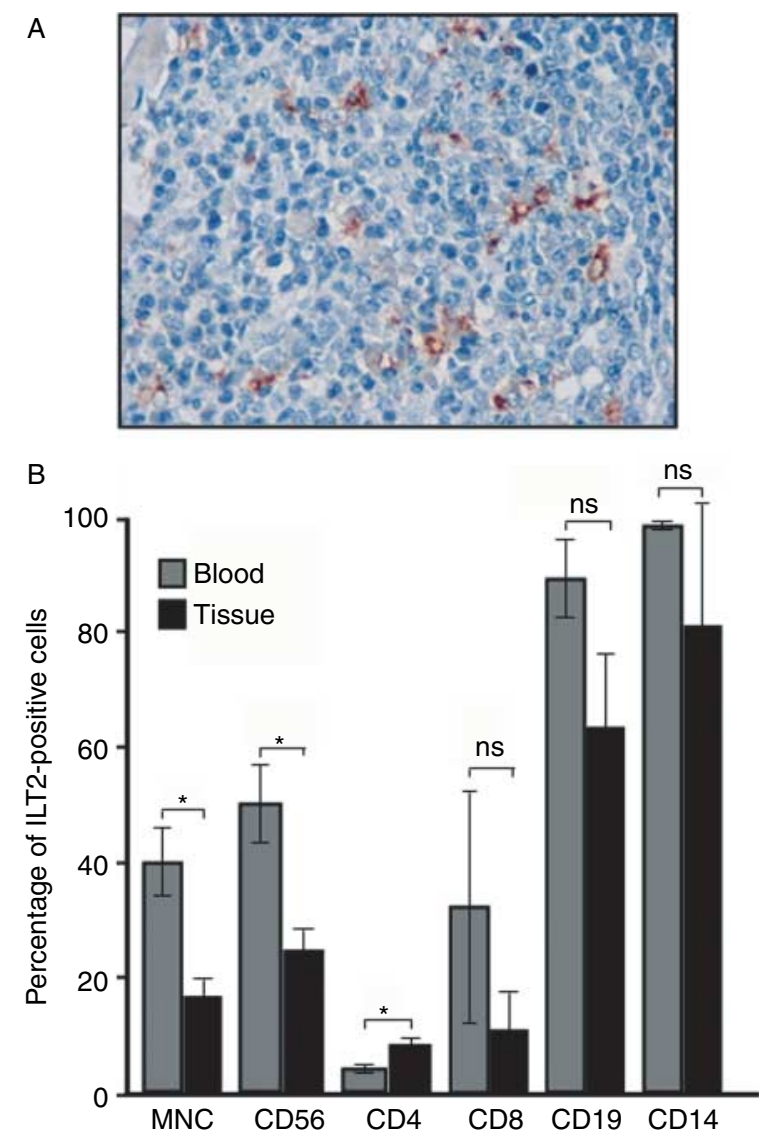

Figure 2 Expression of ILT2 in thyroid glands from AITD patients. (A) Sections of thyroid gland specimens were immunostained for ILT2 and then counterstained with Carazzi's hematoxylin. A representative image of a patient with $\mathrm{HT}$ is shown. Original magnification $400 \times$. (B) Mononuclear cells were isolated from the peripheral blood and thyroid tissue specimens and cells were analyzed by two-color flow cytometry for the expression of ILT2 by whole MNC, CD56+, CD4+, CD8+, CD19+, and CD14+ cells. Data correspond to the arithmetic mean and s.D. ${ }^{*} P<0.05$. ns, nonsignificant; HT, Hashimoto's thyroiditis; GD, Graves' disease. 


\section{In vitro cytokine production assays}

PBMC $\left(1.0 \times 10^{6}\right)$ were cultured, as above, for $48 \mathrm{~h}$ in flat bottom 96-well plate previously coated with anti-CD3/CD28 MAB, in the presence or absence of anti-ILT2 MAB. Then, cell culture supernatants were collected and the concentrations of IL2 and IL10 were determined by ELISA kit assays (Diaclone, Besancon, France) according to the manufacturer's recommendations.

\section{Statistical analysis}

Results were expressed as the arithmetic mean \pm S.D. Statistical analysis was performed the GraphPad InStat 3 program (GraphPad Software, San Diego, CA, USA). Parametric analysis was performed Student's $t$-test and one-way ANOVA. The Mann-Whitney $U$ test and Kruskal-Wallis analyses were used when data were not normally distributed. The analysis of correlations between variables was based on Spearman's or Pearson's tests. Values of $P<0.05$ were considered statistically significant.

\section{Results}

\section{Expression of ILT2 by PBMC from AITD patients}

We first analyzed the expression of ILT2 in different cell populations from the peripheral blood of AITD patients and healthy subjects. Flow cytometry analysis showed a significantly higher expression of ILT 2 in the CD $4+$ and CD8 + T lymphocyte subsets as well as in CD56+ NK cells from HT and GD patients compared with controls $(P<0.05$ in all cases, Fig. $1 \mathrm{~A}-\mathrm{C})$. On the contrary, the levels of expression of ILT2 in B cells $(\mathrm{CD} 19+)$ and monocytes $(\mathrm{CD} 14+)$ were not different in the three groups studied $(P>0.05$ in all cases, data not shown). Interestingly, in patients with HT there was a significant positive correlation between the titers of anti-Tg antibodies and the percent of ILT $2+/ \mathrm{CD} 4+$ cells ( $r=0.63, P<0.01$, Fig. 1E). However, no significant correlations were detected between the level of expression of ILT2 and duration of disease, clinical features, or therapy (data not shown). In this regard, although a nonsignificant association was detected between the levels of CD4+ILT2 + lymphocytes and the titers of anti-TPO antibodies $(r=0.29, P=0.061)$, we consider that it is feasible that by increasing the number of patients studied, a significant correlation might be reached.

\section{Expression of ILT2 in thyroid glands from AITD patients}

Immunohistochemistry analysis of HT thyroid glands showed a scattered but significant proportion of ILT2 + cells (Fig. 2A). Flow cytometry analysis of TMC
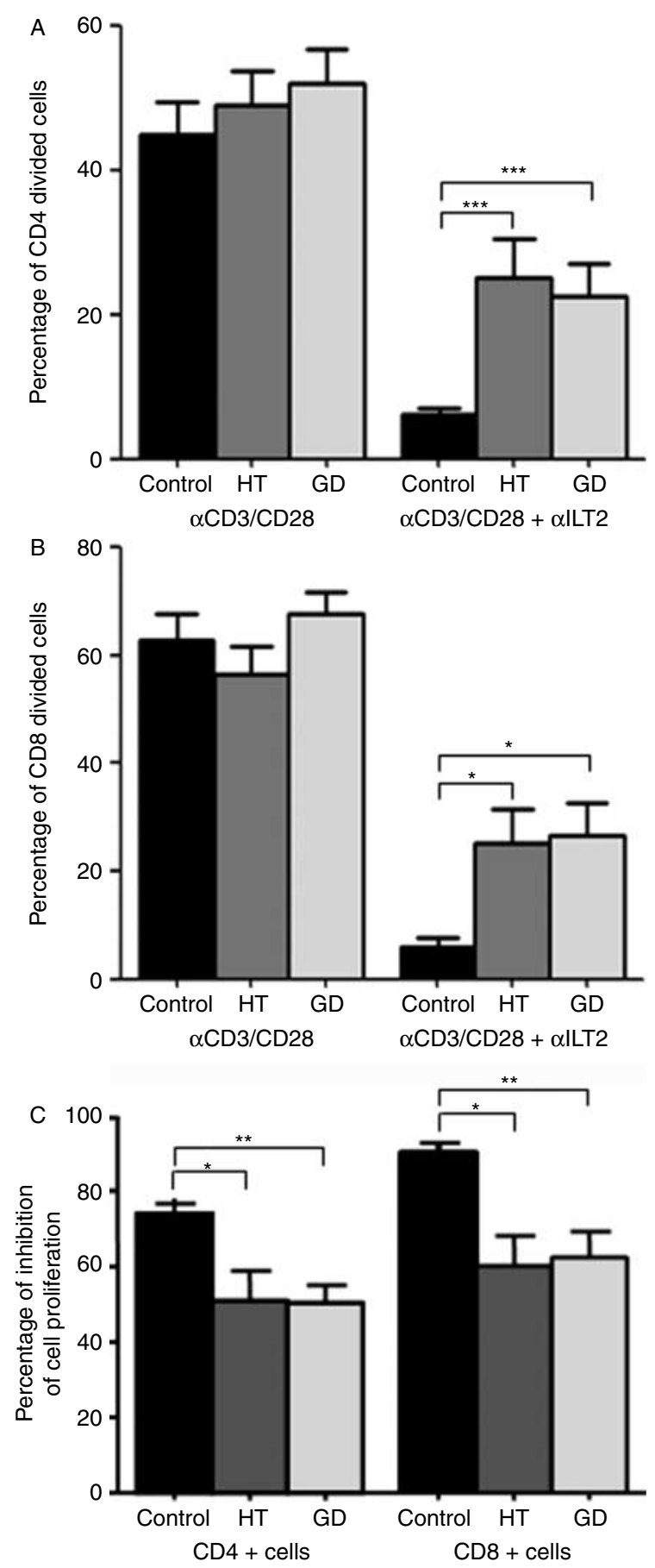

Figure 3 Regulatory effect of ILT2 on lymphocyte proliferation in patients with AITD. (A and B) PBMC were loaded with CFSE and stimulated with anti-CD3/CD28 MAB. Then, ILT2 was engaged or not and cross-linked as stated in Materials and methods section. Finally, cells were cultured for $72 \mathrm{~h}$, labeled for CD8 and CD4, and the percent of divided cells was determined by flow cytometry analysis. (C) Results of the assays shown in panels $A$ and $B$ are expressed as the percent of inhibition of cell proliferation. Data correspond to the arithmetic mean and S.E.M. of the percent of divided cells. ${ }^{*} P<0.05,{ }^{* *} P<0.01,{ }^{* *} P<0.001$. HT, Hashimoto's thyroiditis; GD, Graves' disease. 
confirmed the presence of ILT2 + cells, with enhanced levels of CD4+ILT2 + lymphocytes compared with peripheral blood (Fig. 2B). However, the proportion of MNC and CD56 + cells expressing ILT2 was higher in peripheral blood compared with thyroid tissue (Fig. 2B). No significant differences were detected in the case of NK cells $(\mathrm{CD} 56+)$, B lymphocytes $(\mathrm{CD} 19+)$, or monocytes $(\mathrm{CD} 14+)$.

\section{Inhibitory function of ILT2 in AITD patients}

To further explore the possible role of ILT2 in the pathogenesis of AITD, we tested the suppressive function of this receptor on the proliferation of PBMC from patients with HT, GD, or healthy controls stimulated through $\mathrm{CD} 3$ and $\mathrm{CD} 28$. As shown in Fig. 3A and B, in the absence of ILT2 engagement, a similar level of $\mathrm{CD} 4+$ or CD8 + lymphocyte proliferation was observed in patients with AITD and healthy subjects. As expected, on ILT2 engagement, a significant reduction in cell proliferation was observed in patients and controls (Fig. 3A and B). However, the degree of inhibition of cell proliferation was significantly higher in lymphocytes from healthy controls compared with HT and GD patients, in both, CD8 + and CD4 + cell subsets. When data were expressed as the percent of inhibition of cell proliferation, similar results were obtained (Fig. 3C). Finally, no significant correlations were detected between the degree of the suppressive function of ILT2 and clinical parameters, therapy, or levels of thyroid hormones (data not shown).

The suppressive function of ILT2 in lymphocytes was also assessed by analyzing their progression in cell cycle induced through CD3/CD28 by a flow cytometry DNA content assay. We found that ILT2 engagement induced a significant increase in the levels of hypodiploid apoptotic cells in lymphocytes from healthy controls but not in those from HT or GD patients (Fig. 4A). On the contrary, on ILT2 engagement, a diminution in the number of cells in the $\mathrm{S}$ and $\mathrm{G} 2 / \mathrm{M}$ of cell cycle was detected in healthy subjects but not in AITD patients (Fig. 4B and C).

\section{Regulatory function of ILT2 on the synthesis of cytokines in AITD patients}

IL2 plays an important role in the proliferation of T lymphocytes whereas IL10 mainly exerts an antiinflammatory function and a down-regulatory effect on the cellular immune response (25). Therefore, we decided to evaluate the levels of these cytokines in culture supernatants of cells from patients with AITD. We found that ILT2 engagement resulted in a significant increase in the production of IL10 in healthy controls and AITD patients $(P<0.05$ in all cases, Fig. 5). However, cells from patients with HT and GD showed a significantly lower synthesis of IL10 than lymphocytes
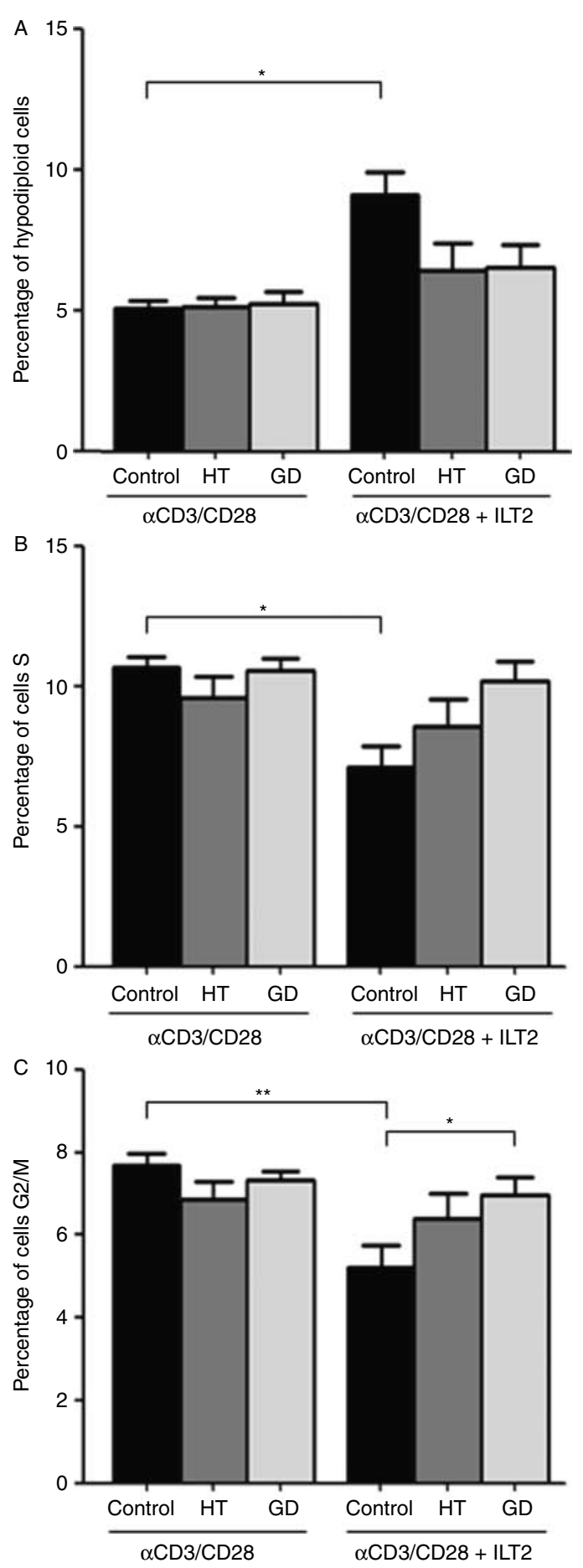

Figure 4 Regulatory effect of ILT2 on cell cycle progression of lymphocytes from AITD patients. PBMC were stimulated with antiCD3/CD28 MAB and then ILT2 was engaged or not and crosslinked as stated in materials and methods section. Finally, cells were cultured for $72 \mathrm{~h}$, stained with propidium iodide and analyzed for DNA content by flow cytometry. Data of hypodiploid cells $(A)$ as well as cells in the $S(B)$ and $G 2 / M(C)$ phases of cell cycle are shown. Data correspond to the arithmetic mean and S.E.M. of cells in the indicated phases of cell cycle. ${ }^{\star} P<0.05,{ }^{\star}{ }^{\star} P<0.01$. HT, Hashimoto's thyroiditis; GD, Graves' disease. 


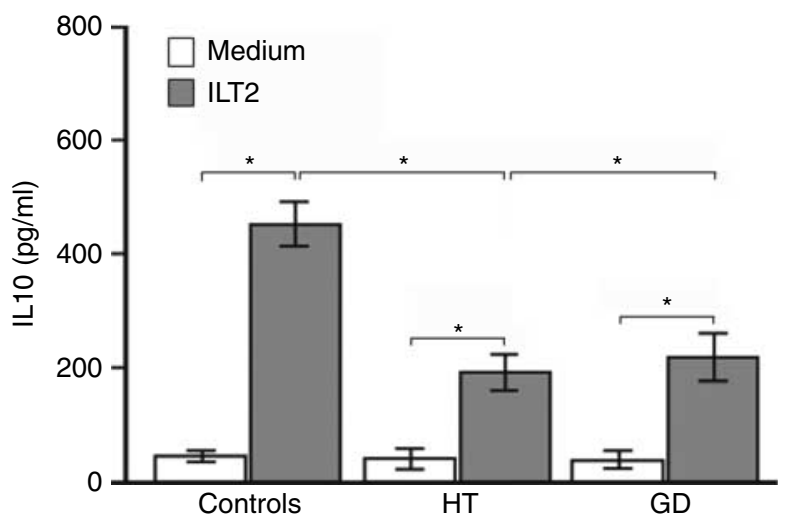

Figure 5 Regulatory effect of ILT2 on the in vitro production of IL10 by lymphocytes from AITD patients. PBMC were stimulated or not with anti-CD3/CD28 MAB, and ILT2 was engaged and cross-linked, as stated in materials and methods section. After $72 \mathrm{~h}$ of cell culture, supernatants were obtained and the levels of IL10 were determined by ELISA. Data correspond to the arithmetic mean and S.E.M. of IL10 concentration. * $P<0.05$. HT, Hashimoto's thyroiditis; GD, Graves' disease.

from healthy controls $(P<0.05$ in both cases). On the contrary, the synthesis of IL2 on ILT2 engagement was not significantly different in the three groups studied (data not shown).

\section{Discussion}

Different receptors have been described to negatively regulate immune responses by bearing inhibitory motifs (ITIMs) in their cytoplasmic tails, including the members of the Ig superfamily, implicated in both innate and adaptive immunity, and known as Ig-like transcripts or ILTs (25). Alterations in the function of these regulatory mechanisms have been associated with the development of inflammatory disorders, autoimmune diseases, and lymphoid malignancies (26). In this regard, previous studies have suggested that ILT receptors are important in the regulation of the immune response in different conditions including systemic lupus erythematosus and rheumatoid arthritis $(4,24,27)$. However, the possible role of ILT2 has not been previously explored in patients with thyroid autoimmunity.

In this study, we investigated the expression and function of ILT2 in patients with HT and GD. We found an unexpected and significant increase in the expression of ILT2 in the PBMC from patients with AITD. In this regard, we have previously described a decreased expression of this regulatory receptor in patients with systemic lupus erythematosus (SLE) (24). Although we do not have a commendable explanation for these contrasting results, it is of interest that Kuroki et al. (27), described two single nucleotide polymorphisms (SNPs) in the promoter region of ILT2 gene, which were associated with rheumatoid arthritis and a diminished synthesis of the protein. Therefore, we consider that it would be of interest to determine the possible presence of SNPs in the ILT2 gene that would cause an enhanced synthesis of the protein, and their possible association with AITD.

We have also detected the expression of ILT2 in the thyroid gland. Our data show significant differences in the proportion of ILT2 + cell subsets when peripheral blood was compared with TMC, with an enhanced number of CD4+ILT2 + lymphocytes in the thyroid glands from AITD patients. These data suggest that there is a selective recruitment of this lymphocyte subset into the thyroid tissue. In this regard, we have previously demonstrated an enhanced proportion of certain lymphocyte subsets in thyroid glands from AITD patients, mainly CD $4+$ GITR + and CD $69+\mathrm{CD} 25^{\text {bright }}$ Foxp3 + cells (17), which might also include ILT2 + lymphocytes. However, these lymphocytes seem to exert a defective regulatory function as discussed below.

We performed an extensive analysis in AITD patients on the possible association between clinical, laboratory, or therapeutic features and the levels of ILT2 + cells, in peripheral blood or thyroid tissue. However, we only detected a significant positive association between the percentage of CD4+ILT2 + lymphocytes and anti-Tg antibodies as well as an apparent correlation with antiTPO antibodies. This phenomenon could be related to the defective function of ILT2 in these patients as discussed below.

Despite the increased levels of ILT2 + cells in their peripheral blood, AITD patients show a defective regulatory function of this receptor on the lymphocyte proliferation induced through $\mathrm{CD} 3 / \mathrm{CD} 28$. As we have previously detected a similar defective function in patients with systemic lupus erythematosus (24), it is very likely that, as in the case of Treg cells (28), this could be a common feature in autoimmune diseases. Therefore, it is possible to speculate that the diminished regulatory function of ILT2 may significantly contribute to the loss of tolerance to self-thyroid antigens, and to the poor control of the inflammatory and destructive phenomenon observed in HT. Thus, AITD patients show a defective function in different immunoregulatory phenomena including those mediated by ILT2 and Treg lymphocytes (17).

As it has been described that the regulatory function of ILT2 may be mediated by the induction of programmed cell death (8), we determined the possible induction of apoptosis on ILT2 engagement. Interestingly, our data indicate that the lymphocytes from AITD patients show a diminished percent of hypodiploid cells when ILT2 is engaged and cross-linked. These data suggest that the defective function of ILT2 in AITD seems to be a consequence, at least in part, of its diminished capability to induce apoptosis. In this regard, it has widely been described the important effect of the dysfunction or absence of death receptors on the 
pathogenesis of autoimmune diseases $(29,30)$. On the other hand, the enhanced proportion of lymphocytes from AITD patients in the $\mathrm{S}$ and $\mathrm{G} 2 / \mathrm{M}$ phases of cell cycle on stimulation and ILT2 engagement is in agreement with the results of cell proliferation assays and further support the defective regulatory function of ILT2 in these patients.

Our assays of cytokine synthesis suggest that the regulatory function of ILT2 is also mediated by a strong induction of synthesis of the anti-inflammatory cytokine IL10. These assays also show that this function is defective in patients with HT and GD. Based on these results, it is possible to speculate that the diminished synthesis of IL10 would favor the generation of the strong cell-mediated autoimmune response observed in HT. However, in the case of GD, the possible consequences of a diminished production of IL10 remain as an interesting point to be defined.

In summary, this study provides evidence that patients with AITD show an abnormal expression and function of ILT2, a phenomena that could significantly participate in the pathogenesis of thyroid autoimmunity.

\section{Declaration of interest}

The authors declare that there is no conflict of interest that could be perceived as prejudicing the impartiality of the research reported.

\section{Funding}

This study was supported by the grant 95395 from the Fondo de Cooperación Internacional de Ciencia y Tecnología (FONCICYT, CONACYT-European Union, México, to R González-Amaro). L DonízPadilla was recipient of a scholarship from CONACYT (México).

\section{Acknowledgements}

We extend special thanks to Dr Eduardo Larrañaga and Dr José Miguel Bravo for their provision of thyroid specimens. We also thank Dr Manuel Luque and Dr Alfonso Arranz for their help in the recruitment and follow-up of the patients.

\section{References}

1 Frauwith KA \& Thompson CB. Activation and inhibition of lymphocytes by costimulation. Journal of Clinical Investigation 2002 109 295-299. (doi:10.1172/JCI14941)

2 Saverino D, Tenca C, Zarcone D, Merlo A, Megiovanni AM, Valle MT, Manca F, Grossi CE \& Ciccone E. CTLA-4 (CD152) inhibits the specific lysis mediated by human cytolytic $\mathrm{T}$ lymphocytes in a clonally distributed fashion. Journal of Immunology 1999162 651-658.

3 Alegre ML, Frauwirth KA \& Thompson CB. T-cell regulation by CD28 and CTLA-4. Nature Reviews. Immunology $20011220-228$. (doi:10.1038/35105024)

4 Brown D, Trowsdale J \& Allen R. The LILR family: modulators of innate and adaptative immune pathways in health and disease. Tissue Antigens 200464 215-225. (doi:10.1111/j.0001-2815. 2004.00290.x)

5 Saverino D, Fabbi M, Ghiotto F, Merlo A, Bruno S, Zarcone D, Tenca C, Tiso M, Santoro G, Anastasi G, Cosman D, Grossi CE \&
Ciccone E. The CD85/LIR-1/ILT2 inhibitory receptor is expressed by all human $\mathrm{T}$ lymphocytes and down-regulates their functions. Journal of Immunology $20001653742-3755$.

6 Cosman D, Fanger N, Borges L, Kubin M, Chin W, Peterson L \& Hsu ML. A novel immunoglobulin superfamily receptor for cellular and viral MHC class I molecules. Immunity 19977 273-282. (doi:10.1016/S1074-7613(00)80529-4)

7 Colonna M, Navarro F, Bellon T, Llano M, Garcia P, Samaridis J, Angman L, Cella M \& Lopez-Botet M. A common inhibitory receptor for major histocompatibility complex class I molecules on human lymphoid and myelomonocytic cells. Journal of Experimental Medicine $1997 \mathbf{1 8 6}$ 1809-1818. (doi:10.1084/ jem.186.11.1809)

8 Saverino D, Merlo A, Bruno S, Pistoia V, Grossi CE \& Ciccone E. Dual effect of CD85/leukocyte Ig-like receptor-1/Ig-like transcript 2 and CD152 (CTLA-4) on cytokine production by antigenstimulated human T cells. Journal of Immunology $2002 \mathbf{1 6 8}$ 207-215.

9 Navarro F, Llano M, Bellon T, Colonna M, Geraghty DE \& LopezBotet M. The ILT2(LIR1) and CD94/NKG2A NK cell receptors respectively recognize HLA-G1 and HLA-E molecules co-expressed on target cells. European Journal of Immunology 199929 277-283. (doi:10.1002/(SICI)1521-4141(199901)29:01 <277::AID-IMM $\mathrm{U} 277>3.0 . \mathrm{CO} ; 2-4)$

10 Colonna M, Nakajima H, Navarro F \& Lopez-Botet M. A novel family of Ig-like receptors for HLA class I molecules that modulate function of lymphoid and myeloid cells. Journal of Leukocyte Biology 199966 375-381.

11 Pearce EN, Farwell AP \& Braverman LE. Thyroiditis. New England Journal of Medicine $2003 \mathbf{3 4 8}$ 2646-2655. (doi:10.1056/ NEJMra021194)

12 Anderson MS. Autoimmune endocrine disease. Current Opinion in Immunology 200214 760-764. (doi:10.1016/S0952-7915(02) 00405-3)

13 Weetman AP. Autoimmune thyroid disease: propagation and progression. European Journal of Endocrinology 2003148 1-9. (doi:10.1530/eje.0.1480001)

14 Ban Y \& Tomer Y. Susceptibility genes in thyroid autoimmunity. Clinical and Developmental Immunology 200512 47-58. (doi:10. 1080/17402520400008897)

15 Ban Y, Tozaki T, Taniyama M, Tomita M \& Ban Y. Association of a CTLA-4 3' untranslated region (CT60) single nucleotide polymorphism with autoimmune thyroid disease in the Japanese population. Autoimmunity 200538 151-153. (doi:10.1080/ 08916930500050319)

16 Figueroa-Vega N, Alfonso-Pérez M, Benedicto I, Sánchez-Madrid F, González-Amaro R \& Marazuela M. Increased circulating proinflammatory cytokines and Th17 lymphocytes in Hashimoto's thyroiditis. Journal of Clinical Endocrinology and Metabolism 2010 95 953-962. (doi:10.1210/jc.2009-1719)

17 Marazuela M, García-López MA, Figueroa-Vega N, De la Fuente H, Alvarado-Sánchez B, Monsiváis-Urenda A, Sánchez-Madrid F \& González-Amaro R. Regulatory T cells in human autoimmune thyroid disease. Journal of Clinical Endocrinology and Metabolism 200691 3639-3646. (doi:10.1210/jc.2005-2337)

18 Figueroa-Vega N, Alfonso-Pérez M, Cuesta-Mateos C, SánchezMadrid F, Moreno-Otero R, González-Amaro R \& Marazuela M. Tie-2 is overexpressed by monocytes in autoimmune thyroid disorders and participates in their recruitment to the thyroid gland. Journal of Clinical Endocrinology and Metabolism 200994 2626-2633. (doi:10.1210/jc.2009-0220)

19 Figueroa-Vega N, Sanz-Cameno P, Moreno-Otero R, SánchezMadrid F, González-Amaro R \& Marazuela M. Serum levels of angiogenic molecules in autoinmune thyroid diseases and their correlation with laboratory and clinical features. Journal of Clinical Endocrinology and Metabolism 200994 1145-1153. (doi:10. 1210/jc.2008-1571)

20 Caturegli P, Kimura H, Rocchi R \& Rose NR. Autoimmune thyroid diseases. Current Opinion in Rheumatology 200719 44-48. (doi:10.1097/BOR.0b013e3280113d1a) 
21 Devendra D \& Eisenbarth GS. Immunologic endocrine disorders. Journal of Allergy and Clinical Immunology 2003111 S624-S636. (doi:10.1067/mai.2003.81)

22 Merlo A, Saverino D, Tenca C, Grossi CE, Bruno S \& Ciccone E. CD85/LIR-1/ILT2 and CD152 (cytotoxic T lymphocyte antigen 4) inhibitory molecules down-regulate the cytolytic activity of human CD4+T-cell clones specific for Mycobacterium tuberculosis. Infection and Immunity 200169 6022-6029. (doi:10.1128/IAI. 69.10.6022-6029.2001)

23 Ince MN, Harnisch B, Xu Z, Lee SK, Lange C, Moretta L, Lederman M \& Lieberman J. Increased expression of the natural killer cell inhibitory receptor CD85j/ILT2 on antigen-specific effector CD8 T cells and its impact on CD8 T-cell function. Immunology 2004112 531-542. (doi:10.1046/j.1365-2567. 2004.01907.x)

24 Monsiváis-Urenda A, Niño-Moreno P, Abud Mendoza C, Baranda L, Layseca-Espinosa E, López-Botet M \& GonzálezAmaro R. Analysis of expression and function of the inhibitory receptor ILT2 (CD85j/LILRB1/LIR-1) in peripheral blood mononuclear cells from patients with systemic lupus erythematosus (SLE). Journal of Autoimmunity 200729 97-105. (doi:10.1016/j. jaut.2007.05.003)

25 Long EO. Regulation of immune responses through inhibitory receptors. Annual Review of Immunology 199917 875-904. (doi:10.1146/annurev.immunol.17.1.875)
26 Veillette A, Latour S \& Davidson D. Negative regulation of immunoreceptor signaling. Annual Review of Immunology 200220 669-707. (doi:10.1146/annurev.immunol.20.081501.130710)

27 Kuroki K, Tsuchiya N, Shiroishi M, Rasubala L, Yamashita Y, Matsuta K, Fukazawa T, Kusaoi M, Murakami Y, Takiguchi M, Juji T, Hashimoto H, Kohda D, Maenaka K \& Tokunaga K. Extensive polymorphisms of LILRB1 (ILT2, LIR1) and their association with HLA-DRB1 shared epitope negative rheumatoid arthritis. Human Molecular Genetics 200514 2469-2480. (doi:10. 1093/hmg/ddi247)

28 Alvarado-Sánchez B, Hernández-Castro B, Portales-Pérez D, Baranda L, Layseca-Espinosa E, Abud-Mendoza C, CubillasTejeda AC \& González-Amaro R. Regulatory T cells in patients with systemic lupus erythematosus. Journal of Autoimmunity 2006 27 110-118. (doi:10.1016/j.jaut.2006.06.005)

29 Stassi G \& De Maria R. Autoimmune thyroid disease: new models of cell death in autoimmunity. Nature Reviews. Immunology 20022 195-204. (doi:10.1038/nri750)

30 Mahoney JA \& Rosen A. Apoptosis and autoimmunity. Current Opinion in Immunology 200517 583-588. (doi:10.1016/j.coi. 2005.09.018)

Received 8 April 2011

Accepted 4 May 2011 